Fecha de recepción: diciembre 2010

Fecha de aceptación: julio 2011

Versión final: marzo 2012

\section{Una guía comentada acerca de la tipología y la morfología de Pierre Schaeffer}

Claudio Eiriz ${ }^{\star}$

\begin{abstract}
Resumen: La publicación del presente artículo tiene como propósito facilitar la comprensión de la Tipología y la Morfología de los objetos sonoros, incluida en el célebre Tratado de los objetos musicales de Pierre Schaeffer.

En primer lugar, se describe la fase exploratoria de la construcción de la taxonomía de los sonidos, que Schaeffer denominó Tipo-Morfología. En segundo lugar, se presentan los nueve casos de Tipología Central. Y, por último, se explican los siete criterios morfológicos.
\end{abstract}

Palabras clave: morfología - objetos sonoros - tipología.

[Resúmenes en inglés y portugués en la página 56]

${ }^{(*)}$ Licenciado en Ciencias de la Educación, además de psicopedagogo (UNLZ). Postgraduado en Semiología de la Música (UBA). Cursó estudios de "Percusión" con los maestros Orlando Giacobbe y Alfredo Suárez en el (IMMA y EMPA). Docente de la Facultad de Diseño y Comunicación de la Universidad de Palermo.

\title{
Introducción
}

La introducción del libro Una voz y nada más de Mladen Dolar se inicia con el siguiente chiste:

En medio de una batalla un Comandante del ejército italiano da la siguiente orden: 'soldados ¡ataquen!' Los soldados, que están atrincherados, no atinan a moverse. El comandante emite por segunda vez la orden y nuevamente, nadie se mueve. Intenta una tercera vez y uno de los soldados exclama 'Che Bella voce' (ique bella voz!) (2007, p.13).

Está claro que los reclutas no se reconocen en su misión en tanto soldados en una batalla. Y es por ello que no comprenden la orden del comandante. Sin embargo la respuesta elogiosa del soldado nos da pruebas de que si la interpelación del comandante fue fallida es porque sólo pudieron reconocerse como destinatarios de otro mensaje. Actúan como la comunidad de personas que aprecia la estética del bel canto; como buenos italianos amantes de la ópera.

Es indudable que los soldados se centraron en la voz y no en el mensaje.

Este ejemplo puede explicar muy bien el sentido de las celebres "Tres escuchas" que teorizó 
Pierre Schaeffer en su Tratado de los objetos musicales (1988, pp. 61-74). Para ser sintético diré que, según Schaeffer, habría tres posiciones de escucha.

En la escucha causal el sonido funciona como un índice. El oído se dirige a identificar la causa que produce el sonido.

En la escucha semántica el oído se dirige a comprender el mensaje En este caso tampoco interesa el sonido en sí mismo. El sonido funciona como soporte material de un sentido. El ejemplo paradigmático es el lenguaje.

En la escucha reducida se toma al sonido como un objeto de observación en si mismo. ${ }^{1}$ No nos interesamos ni por la causa ni por el sentido. Al escuchar en forma reducida nos focalizamos en las cualidades intrínsecas del sonido.

Los soldados italianos del chiste antes comentado no se habían interesado ni por las causas del sonido -el comandante que emitía la voz- ni por el sentido de las palabras. Se interesaron por la voz en si misma.

La voz es el soporte material del significado. Digamos que posibilita el acto mismo del habla pero no contribuye a producir sentido. Es la materia prima en la que se operan las oposiciones diferenciales. Pero son estas oposiciones diferenciales las que importan a la lingüística. El sonido queda allí como un resto un tanto evanescente, que constituye una condición del habla pero que al mismo tiempo parecería estar fuera de ella.

Los soldados italianos anclaron allí, en esa materialidad del sonido de la voz y lo convirtieron en objeto de placer estético que también, a su modo, es portador de un significado, aunque de un orden distinto al del lenguaje.

Sin embargo, es posible anclar en esa materia significante que es el sonido, sin hacerla objeto de culto ni de placer estético. Esta otra alternativa es tomar al sonido mismo como objeto de estudio, más allá del sentido o de las causas.

Convengamos que nada puede funcionar como significante si previamente no es una cosa definida, con sus propiedades. Estudiar las propiedades de esa "cosa" definida, de ese resto que queda por fuera del sentido -y que por otra parte, es la materia prima con la que trabajan los músicos- es a mi juicio uno de los grandes aportes de los trabajos de Pierre Schaeffer.

Tomar al sonido como objeto de estudio mediante la posición de escucha reducida es a lo que Schaeffer denominó objeto sonoro. En este artículo intentaré hacer un resumen de la tipología y la morfología del objeto sonoro. Es decir, la clasificación y la descripción del universo de sonidos en tanto objetos de la escucha reducida. ${ }^{2}$

Dos cosas me animan a publicarlo. La primera es que, hasta donde yo se, no existe en castellano, un trabajo más o menos completo acerca del tema. En el libro El sonido de Michel Chion (1999, pp. 295-350) hay un capítulo denominado "Describir y clasificar sonidos", que aborda esta cuestión, pero está apenas esbozado. Otro libro de Chion Guide des objets sonores (1983), es un resumen muy completo acerca del la obra de Schaeffer, pero no hay -hasta donde llega mi conocimiento- traducción al castellano.

El solfeo del objeto sonoro publicado por Schaeffer y colaboradores (1998) se detiene en la tipología, pero los aspectos morfológicos quedan un tanto diluidos. ${ }^{3}$

La segunda razón que me anima a publicar este artículo es que la tipo-morfología schaffereana ha tenido gran impacto en la enseñanza del audiovisual de la mano de Michel Chión en Francia (1993), y de Carmelo Saitta, en Argentina (2002). Este hecho justifica el tratamiento de este tema en una publicación de una Facultad de Diseño y comunicación. 
El Trabajo de Schaeffer debe ser entendido, entre otras cosas, como una herramienta para enriquecer la percepción sonora. Es posible realizar una suerte de aplicación pedagógica de la tipología y la morfología. Beatriz Ferreyra, en el prefacio al Solfeo del objeto sonoro escribe, "La clasificación del solfeo no es en sí una finalidad, sino una herramienta de trabajo que afina, amasa y hace consciente, la percepción de lo sonoro y por extensión, la percepción del fenómeno musical" (Schaeffer y colaboradores, 1998).

En un sentido similar, Michel Chion plantea, "Nuestra hipótesis es que la exigencia de precisión verbal activa es un medio primordial de afinación y cultivo de la percepción” (1999, p. 378).

Así mismo, Delalande, en su libro de pedagogía La música es un juego de niños, titula un capítulo con el nombre de "Palabras para describir sonidos". Allí nos dice que si nuestro vocabulario para describir sonidos es acotado escuchamos pobremente, en otras palabras, necesitamos palabras para escuchar. Más adelante agrega:

En el comienzo de la música concreta Schaeffer se encontró con colecciones de ruidos grabados, con los cuales quería hacer música y confrontado a un problema de clasificación y de descripción. Entonces buscó palabras que permitieran analizar todos los sonidos (...) (Delalande, p. 58).

Es claro que en estas tres citas existe algo en común: la enseñanza de los trabajos schaeffereanos, puede ser entendida como un medio para maximizar la escucha.

Para dar una prueba más que apoye esta hipótesis de derivación pedagógica del Tratado de Schaeffer, vayamos a su post scriptum, que concluye con una cita que su autor hace de Descartes.

que si quieren seguir un designio parecido al mío, no tienen necesidad de que les diga más de lo que ya he dicho en este discurso, pues si son capaces de ir más allá de lo que yo he hecho, con más razón lo serán para encontrar por sí mismos todo lo que creo haber encontrado...

\section{La Tipomorfología}

El programa de investigación acerca del objeto sonoro, llevado a cabo por Pierre Schaeffer, puede ser entendido como un intento de construir un aparato de categorías destinado a clasificary describir la totalidad de los sonidos. Para ello fue necesario, en principio, la consecución de tres objetivos: el primero referido a la necesidad de identificación de las unidades sonoras fundamentales; el segundo, a la clasificación de esas unidades en tipos; y el tercero, a una permanente búsqueda de criterios, cada vez más sutiles, de descripción.

Schaeffer denominó "Tipología" al trabajo de identificación y clasificación de los sonidos; y "Morfología" al trabajo de descripción de los sonidos en su contextura interna.

Este ambicioso programa se inició con una primera fase exploratoria denominada Tipo- Morfología. En esta fase inicial del trabajo las tres operaciones (identificar, clasificar, describir) ${ }^{4}$, se fueron realizando en forma conjunta y por aproximaciones sucesivas.

Imaginemos la situación: Schaeffer se encontró con un campo no explorado; no había antece- 
dentes de estudios del sonido desde la escucha reducida; nadie, hasta donde yo se, había planteado el sonido como objeto de observación en esos términos. O en otras palabras, Schaeffer estaba construyendo su objeto de estudio, bastante original en ese momento.

Diseñar un objeto de investigación implica, entre otras cosas, decidir cuáles entidades del campo de estudio van a ser consideradas unidades de análisis y cuáles serán los criterios de clasificación y descripción, relevantes para esa investigación.

En virtud de ello, se hizo necesario que Schaeffer comenzara por definir criterios de identificación de unidades sonoras. Es decir, recortar del continuo sonoro, las unidades que serán a su turno, objeto de agrupamiento y descripción.

Para realizar una tipología de unidades sonoras y posteriormente describirlas es preciso, ante todo, delimitar esas unidades. En otras palabras, saber qué cosa vamos a hacer objeto de clasificación y descripción.

La primera operación de la tipología es, como ya he dicho, la identificación de objetos sonoros del continuo sonoro.

Más adelante entraré en detalle acerca del problema de la identificación de las unidades. Sólo adelanto que este problema puede ser sintetizado con la siguiente pregunta: ¿Cuáles son los criterios que nos permitirán delimitar las unidades a partir del continuo sonoro?

Una vez acordados los criterios para recortar los sonidos (unidades) del continuo sonoro, se procede a una clasificación de los mismos, es decir a agruparlos por tipos.

Clasificar significa agrupar elementos, formar clases de objetos conforme a determinados criterios. Pero para hacer esto, al mismo tiempo, se hace necesario perfilar algunos criterios relevantes que posibiliten su descripción.

Supongamos que quiero armar una tipología de sillas. En principio no tendré dificultades en distinguirlas de otros objetos (mesas, aparador, etc.) ni distinguir sus componentes (Patas, respaldo etc.), esto es, identificar los objetos a ser clasificados, y los criterios de fragmentación del objeto. Por otra parte, tengo que disponer de criterios que me permitan describir las distintas características de las sillas. Es necesario que establezca algún criterio y comparar las sillas entre sí. Podemos pensar por ejemplo en la forma, en la cantidad de elementos constitutivos, en el modo en que se distribuyen esos elementos en el espacio; el material con que están hechas, etc. Ciertamente estos criterios pueden ser innumerables, razón por la cual se hace imprescindible simplificar ese "espacio de atributos", tomando en cuenta sólo los criterios relevantes en función de los propósitos de la clasificación. Y luego a partir de allí, formar tipos de sillas. Mi propósito puede ser clasificar sillas con el objeto de distribuirlas mejor en un espacio determinado, en consecuencia realizaré la clasificación conforme al tamaño, la forma, posibilidad de ser encastradas etc.

Para clasificar objetos, es decir, saber en qué se parecen y en qué se diferencian, es preciso contar previamente con criterios de descripción. Pero al mismo tiempo para hacer una descripción necesitamos criterios de clasificación. Este es el problema con el que Pierre Schaeffer se enfrentó en el comienzo de su investigación: "La tipología o arte de separar los objetos sonoros y, si es posible hacer con ellos una tosca clasificación inicial, no puede fundarse más que a partir de rasgos morfológicos" (Schaeffer, 1988, p. 222).

Se hizo necesario, entonces, disponer como mínimo de una rudimentaria morfología; seleccionar unos criterios que permitan decidir en qué medida esos objetos se asemejan o se diferencian, "Por ello, durante muchos años, hemos basculado entre una morfología apenas formula- 
da y una tipología mal definida (Schaeffer, 1988, p. 219)."

Otorgarle un lugar en el seno de una clase a determinada unidad sonora, implica haber elaborado criterios de descripción. En otras palabras, delimitar un elemento de un universo de estudio implica determinar cuáles son los atributos o cualidades que debe reunir para que pueda ser incluido en una clase.

\subsection{Identificación de los objetos sonoros: tipología elemental}

\section{La pareja Articulación / Apoyo}

En esta fase de la investigación, y en dirección a realizar un primer esbozo de clasificación, la operación ha sido la de identificar las unidades de análisis. Esto es, elegir algunos criterios para extraer del continuo sonoro las unidades mínimas.

"La suma del mundo sonoro es apabullante" dice Schaeffer (1988, p. 219). Cosa que obliga a buscar una regla de identificación, es decir algún criterio que se pueda aplicar, aunque sea provisoriamente, a todas las cadenas sonoras.

Las cadenas sonoras son, para Schaeffer, el lenguaje humano, el lenguaje animal, la música y el ruido.

En cada una de estas cadenas es fácil delimitar sus unidades de manera más o menos espontánea. Para el lenguaje hablado, la palabra; para el canto del pájaro, el trino; para la música la nota, el motivo; para el ruido, el conjunto de indicios que permiten reconocer su causa.

Ahora bien, a esos objetos sonoros que se les ha aplicado una "drástica regla de identificación", que han sido aislados de manera más o menos convencional, se les aplica un segundo criterio. Veremos cómo:

Dejando de lado el origen de esos sonidos y su significado, y tomando como recurso a la fonética, aunque forzando un poco los términos, Schaeffer se va a servir de los conceptos de articulación y apoyo.

Con respecto a la articulación, podemos decir que existen sonidos articulados tales como las sílabas del lenguaje y otros inarticulados como un torrente, el sonido continuo de un río, entre otros. Como esta clasificación tiene como propósito al objeto musical y no al lenguaje, Schaeffer va a dejar de lado el concepto de articulación y en su lugar definirá el mantenimiento, es decir la energía aportada en el momento en que "atacamos" el sonido y que se puede comunicar de manera inmediata o prolongada. Notemos que, en última instancia, la manera de atacar un sonido está vinculada de modo análogo a la articulación en la fonética.

El concepto de apoyo, en fonética, se refiere al color de las vocales y en menor medida a la entonación. Como el proyecto es hacer una clasificación de sonidos en dirección a propósitos musicales, es más apropiado hacer hincapié en la entonación. El concepto de entonación plantea la pregunta acerca de si un sonido está fijo o no en la tesitura. De hecho la palabra apoyo sugiere fijeza, en cambio entonación implica también variaciones de altura. De todos modos, en ambos casos sugiere un fenómeno sonoro que se prolonga (Chion, 1983, pp. 114).

En resumen, digamos que la pareja articulación -apoyo tomada de la fonética se transforma en la pareja mantenimiento- entonación cuando la intención es orientada en dirección a propósitos musicales.

Ahora bien, si aplicamos esta pareja mantenimiento- entonación a las unidades de las distintas 
cadenas, obtenemos los siguientes casos:

Mantenimiento prolongado - entonación fija. (Una nota larga de violín que mantiene su altura) Mantenimiento prolongado - entonación variable. (Un glisssando de flauta en émbolo) Mantenimiento nulo - entonación fija. (Un nota de vibráfono)

Mantenimiento nulo - entonación variable. (Una percusión de timbal, desplazando el pedal) ${ }^{5}$

\subsection{Descripción elemental del objeto sonoro: morfología elemental}

\section{La pareja Forma / Materia}

Otra operación, es la elección de criterios de descripción más o menos generales.

Así como los criterios de identificación mas elementales de la tipología es la pareja Mantenimiento /Entonación, los criterios que permiten una primera descripción de los objetos sonoros, es la pareja Forma / Materia.

La materia de un sonido es aquello que se perpetúa a través de la duración.

Imaginemos que fuera posible "parar" para oír lo que es, en un instante determinado de nuestra escucha: lo que captamos, escribe Schaeffer, es su materia (Schaeffer, 1988, p. 224).

La forma es la envoltura de la materia; es la manera en que esa materia se desarrolla en la duración. Como se verá más adelante, Schaeffer va a elaborar criterios cada vez más sutiles de descripción. Algunos de estos criterios morfológicos estarán vinculados a la materia y otros a la forma. Por ejemplo los criterios de Masa, Timbre Armónico y Grano pueden ser considerados criterios pertenecientes a la materia del sonido. Los criterios de Marcha (allure) y perfil dinámico pueden ser considerados criterios de descripción de la forma (Chion, 1983, p. 116).

Sin embargo las nociones de forma y de materia no se dejan delimitar tan fácilmente. Hay que tomarlas como herramientas para el análisis del sonido que nos van a obligar a replantear el problema de la descripción sonora, y en consecuencia, aguzar la escucha (Chion, 1999, p. 308). Hasta aquí he resumido y comentado este primer intento de Schaeffer de construir una tipología elemental y una morfología elemental. Es decir aquello que se denomina Tipo-morfología. En adelante intentaré hacer una síntesis de otras dos fases de la construcción de esta taxonomía. Lo que sigue es entonces, tanto una tipología más desarrollada, como así también una morfología más sutil.

\section{Tipología de los objetos sonoros 6}

\subsection{Criterios tipológicos}

\section{Criterios de masa y factura}

La factura es la percepción cualitativa del mantenimiento. Para entender este concepto recordemos que el Mantenimiento es el proceso energético que se mantiene o no en la duración. Según su mantenimiento los sonidos pueden ser de:

Mantenimiento nulo: Es el caso de las percusiones. Cuando percutimos una fuente o pulsamos una cuerda puede suceder que la energía se disipe rápidamente o que tenga una extinción lenta 
pero no mantenemos el sonido.

Mantenimiento continuo: Por ejemplo por frotado o soplido.

Mantenimiento iterativo. Consistente en una repetición rápida de sonidos breves.

La factura en cambio, es la percepción cualitativa del mantenimiento. Está en relación a éste, pero no se confunde con el mismo. El mantenimiento es una noción "neutra" y la factura es un criterio de descripción musical, describe al mantenimiento.

Cabe aquí una aclaración. La noción de factura, como veremos, es pertinente sólo en el caso de que el objeto sea equilibrado, es decir, tenga un tiempo óptimo de memorización y un cierto grado de previsibilidad (Chion, 1983, p. 118). Si un objeto tiene una duración demasiado larga o bien es demasiado imprevisible, no podemos hablar de factura. Hablaremos en ese caso, meramente, de mantenimiento. Cuando un sonido es demasiado largo tendemos a olvidar su factura y la atención se centra en los detalles internos al sonido, o sea, aquello que hemos denominado su materia. Si el sonido es demasiado imprevisible la percepción tampoco se centra en la factura. Se hace muy difícil retener la forma de ese sonido.

Distinguiremos entonces tres tipos de factura:

\section{Impulsiones}

Son breves, un ataque con una resonancia.

El sonido de un Wood Block (cajas chinas), caracterizado por ser muy breve, con un ataque abrupto y una resonancia dada en general por la reverberación de la sala; un Pizz de violín; etc.

\section{Mantenidos y resonancias formadas.}

Ni muy largos ni muy cortos. Tienen una forma cerrada, se percibe en ellos, claramente el ataque, el cuerpo y la extinción. Tienen un tiempo óptimo de memorización y un cierto grado de previsibilidad. Está claro que pueden ser tanto sonidos carentes de mantenimiento, como del tipo percusión- resonancia (Una percusión de un platillo o la nota de un piano), como sonidos mantenidos, como un frotado de cuerda o el sonido de una flauta.

\section{Iteraciones}

Repetición de impulsiones que tienden a ser percibidos como una totalidad. En este caso también se aplica la misma regla acerca del equilibrio del objeto. Por ejemplo un grupo de semicorcheas no necesariamente se escucha como varias impulsiones aisladas. En general lo escuchamos como una totalidad.

\section{Criterio de Masa}

La Masa representa la generalización del concepto de Altura. Es derivada de la noción de entonación. Como habíamos dicho, podemos describir un sonido de acuerdo a la fijeza en la tesitura. Algunos sonidos pueden estar fijos en la tesitura y otros no. Diremos ahora que: lo que varía es su Masa.

La pregunta que se plantea es la siguiente ¿por qué cambiar el término "altura" por el de "Masa"? Los dos conceptos no significan lo mismo. La masa es la generalización del concepto de altura. Que un sonido, por ejemplo, no tenga una altura "definida" no significa que no tenga una Masa. ${ }^{7}$ 
En algunos sonidos podemos identificar una altura precisa y en otros no. Sin embargo, a estos últimos, es posible situarlos en una zona del registro. Este criterio, en principio, está vinculado al de grado de tonicidad de un sonido.

En resumen, un sonido puede tener una Masa Fija o variada y por otra parte, puede ser tónico o complejo. Schaeffer va a retener los siguientes valores que puede adoptar la Masa de un sonido

La Masa de un sonido puede ser:

Tónica. Tienen una altura precisa. Es decir que podemos "cantarla".

Compleja. No tiene una altura definida, aunque es posible situarla en una zona del registro. El sonido característico del platillo o el de una maraca. Este es un concepto muy amplio. Están incluidos en esta categoría, desde los sonidos inarmónicos de componentes discretos hasta las bandas de ruido.

Variada. Significa que el sonido glissa. (que varía su "altura”). El flexatón por ejemplo, es un instrumento que tiene la característica de hacer variar el sonido en altura. La flauta de émbolo también.

\section{Criterio duración - variación}

Está relacionado a la duración del objeto sonoro y a la variación según los criterios de Masa y de Factura.

La duración está referida a la duración percibida y no a la cronométrica. Y variación, como cualquier cosa que cambia en función del tiempo.

Se distinguen duraciones cortas, medias y extendidas; $y$ variaciones nulas, razonables e imprevisibles.

\section{Criterio equilibrio y originalidad}

El equilibrio es un compromiso variable en la factura del objeto entre lo más estructurado y lo simple. "El grado de originalidad es, grosso modo lo que sorprende a la previsión" (Schaeffer, 1988, p. 231).

Estas dos nociones son complementarias.

A los sonidos que tienen un buen compromiso entre originalidad y equilibrio, Schaeffer los denomina objetos convenientes y los va a ubicar entre los objetos denominados Equilibrados. A los objetos que no tienen un buen compromiso entre originalidad y equilibrio: los No Equilibrados los subdivide en dos grupos. El primer grupo reúne a todos aquellos que son de originalidad nula, y los denomina objetos redundantes. Al segundo grupo pertenecen aquellos sonidos cuya originalidad es excesiva, y los denomina objetos excéntricos. 


\subsection{Objetos equilibrados (tipología central)}

Es posible resumir los tipos de sonidos resultantes teniendo en cuenta los criterios de Masa y Factura.

\begin{tabular}{|c|c|c|c|}
\hline $\begin{array}{l}\text { Sonidos } \\
\text { fijos } \\
\text { tónicos }\end{array}$ & $\begin{array}{l}\text { Formados o } \\
\text { Tenidos } \\
\text { N }\end{array}$ & $\begin{array}{l}\text { Impulsión } \\
N^{\prime}\end{array}$ & $\begin{array}{l}\text { Iteraciones } \\
\text { N" }\end{array}$ \\
\hline $\begin{array}{l}\text { Sonidos } \\
\text { fijos } \\
\text { complejos }\end{array}$ & $\begin{array}{l}\text { Formados o } \\
\text { Tenidos } \\
\text { X }\end{array}$ & $\begin{array}{l}\text { Impulsión } \\
X^{\prime}\end{array}$ & $\begin{array}{l}\text { Iteraciones } \\
\text { X" }\end{array}$ \\
\hline $\begin{array}{l}\text { Sonidos } \\
\text { variables }\end{array}$ & $\begin{array}{l}\text { Formados o } \\
\text { Tenidos } \\
\text { Y }\end{array}$ & $\begin{array}{l}\text { Impulsión } \\
Y^{\prime}\end{array}$ & $\begin{array}{l}\text { Iteraciones } \\
\text { Y" }\end{array}$ \\
\hline
\end{tabular}

Tabla 1. Sonidos Resultantes. Fuente: Elaboración propia.

Sonidos Fijos, Tónicos, Impulsivos. (Pizz de violín)

Sonidos Fijos, Complejos, Impulsivos. (Golpe de maraca)

Sonidos Variables, Impulsivos (Gliss breve de instrumento de cuerda)

Sonidos Fijos, Tónicos, Formados (Nota de piano)

Sonidos Fijos, Complejos, Formados. ( golpe de platillo)

Sonidos Variables, Formados. (Gliss de flauta de émbolo)

Sonidos Fijos, Tónicos, Iterados. ( tremolo de instrumento convencional )

Sonidos Fijos, Complejos, Iterados. ( redoble abierto de tambor)

Sonidos Variables, Iterados. (Gliss de marimba)

\subsection{Objetos no equilbrados}

Además de los nueve casos de la tipología central, presentados anteriormente, Schaeffer clasificó más de treinta de tipos de objetos que ubicó en la periferia de su cuadro tipológico. Denominó a estos tipos de objetos "no equilibrados". Y como ya hemos dicho, a su vez distinguió entre Objetos redundantes o poco originales (objetos de duración bastante larga ), por un lado y objetos excéntricos, (objetos con una organización demasiado compleja) por otro.

Como escribe Chion, Schaeffer consideró a los nueve casos de la tipología central como los más propicios para una construcción musical. Sin embargo los objeto No Equilibrados, que Schaeffer relegó a la periferia de su cuadro clasificatorio, fueron finalmente los más utilizados en la música contemporánea y en diseños de sonido en los audiovisuales (Chion, 1999, p. 311). 
En este artículo no entraré en detalles acerca de los objetos no equilibrados. Se hace muy dificultoso describir cada tipo de objetos sin tener el auxilio de ejemplos sonoros. Por otra parte, la complejidad de extensión del tema merece un artículo aparte.

\section{Morfolofía de los objetos sonoros 8}

Schaeffer denomina morfología a la operación de descripción de los objetos sonoros en su contextura interna.

Los criterios son siete. Y serán ordenados en este texto conforme a la materia, la forma, la variación y al mantenimiento, como lo ha hecho M. Chion en su Guía del objeto sonoro (1983).

\subsection{Criterios que describen la materia}

\section{Masa}

Como se ha dicho, la masa es la generalización del concepto de altura. Creo que es conveniente aclarar esta noción. Antes de Schaeffer - y todavía hoy- se decía que los sonidos pueden agruparse en sonidos de altura determinada y sonidos de altura indeterminada. Los sonidos de altura determinada serían pues, todos aquellos sonidos característicos, producidos por los instrumentos tradicionales a excepción de la mayoría de los instrumentos de percusión. Estos últimos, por ejemplo una sonaja o un platillo, serían, según esa clasificación, sonidos de altura indeterminada. Con la misma lógica se ha utilizado otra clasificación que define como sonidos musicales a aquellos que tienen una altura determinada y ruidos a los que no. Para los sonidos del primer tipo (altura determinada o sonidos musicales), tenemos mucho que decir; somos capaces de precisar la zona del registro en que se encuentran situados, y la altura según una escala de valores (do 4, sol 6 etc.). Este tipo de sonidos por lo general, en términos acústicos, corresponden a sonidos de espectro armónico, son bastante parecidos entre sí, (razón por la cual se pone de manifiesto el valor de altura). Dentro de la segunda categoría (altura no determinada o ruidos) se depositan, a diferencia de los anteriores, sonidos de las más variadas características. El uso de esta lógica de clasificación explica por qué los sonidos de un platillo, de un triángulo y de una maraca, siendo indudablemente muy distintos entre sí, hayan coexistido, durante largo tiempo, amontonados en el mismo desván.

Dijimos que según su Masa los sonidos podían ser tónicos o complejos. Fácilmente al enfrentarnos a esta nueva -pero no tan nueva- clasificación, nos vemos tentados a acomodar las nuevas nociones a las viejas lógicas.

Es cierto que lo anterior no es del todo falso, e incluso no está mal para comenzar, pero la noción de masa sería inútil si sólo se definiera de ese modo. Si así fuera, sería una invención de sinónimos innecesaria.

En todo caso, el concepto de masa puede ser, en principio, una herramienta para identificar, o poner de manifiesto, ciertas cualidades del sonido que hasta el momento se han tenido poco en cuenta.

La inclusión de la noción de Masa sólo se justifica si la lógica que sustenta la clasificación es otra. La lógica de sonido - ruido, pone el acento en los sonidos de espectro armónico y les reserva, a 
los no armónicos, apenas un casillero.

Cuando decimos que la Masa es la generalización del concepto de altura, nos referimos a que vamos a tener la intención de percibir qué cosa nos puede decir el sonido acerca de la altura, de qué modo cada sonido (cualquiera sea) ocupa el campo de las alturas; la manera en que se sitúa el sonido en ese campo más allá de sus características espectrales.

La noción de Masa puede ser pensada como a una suerte de "anchura". Los sonidos de masa tónica son algo así como un punto; se los puede situar muy bien (recordemos do4 etc); los sonidos complejos son menos precisos en su situación, pero no por ello dejan de ocupar el campo de las alturas. De hecho se puede reconocer cuál es la ubicación que tiene un sonido complejo en el registro (si es agudo, sobre agudo, grave y demás) y que cantidad de lugar ocupa, es decir si su masa es más o menos estrecha o más o menos ancha. Schaeffer reserva las nociones de Emplazamiento y Calibre para tratar estos temas.

Otra tentación sería acomodar las nociones de masa tónica y masa compleja a las nociones de sonidos de espectro armónico y sonidos de espectro inarmónico y bandas de ruido. Es cierto que en los sonidos de espectro armónico se va a percibir una masa tónica, pero muchos sonidos inarmónicos de componentes discretos también van a ser percibidos con una masa tónica. Para que una masa sea tónica, basta con poder "cantar" el sonido. Osea que "represente un punto" en el campo de las alturas.

Es más, algunos sonidos según el contexto van a funcionar como intervalo o como "color"; tenderán a integrarse a estructuras escalares o a tramas "tímbricas". Pensar la masa de un sonido en términos de anchura nos deja en condiciones de poder describir con mayor sutileza sus características. Otro aspecto que marca una diferencia con la lógica clasificatoria anterior a Schaeffer es la inclusión de la noción de variación en este campo. Es verdad que la noción de glissando forma parte del vocabulario musical desde hace ya tiempo. De todos modos la noción de masa variada en Schaeffer supera los términos restringidos de la noción de glissando y le reserva dos criterios aparte en su morfología que denomina Perfil de masa y Perfil melódico. La Masa puede variar, no sólo en cuanto a su emplazamiento o tesitura, sino también en cuanto a su calibre (su anchura).

\section{Tipos de masa}

En la tipología de las masas Schaeffer va a incluir solo los casos en que la masa está fija. Es decir sonidos tónicos y complejos, fijos en la tesitura. A los sonidos de masa variable les reserva, como ya he mencionado, los criterios de Perfil de masa y perfil melódico.

\section{Clases de masa}

Donde mejor se nos revelan las cualidades de la materia del sonido es en los sonidos homogéneos. Estos tienen una duración tal que permiten escuchar los detalles del cuerpo del sonido. Es común que los investigadores intenten aislar entre los múltiples objetos, aquel que mejor ponga de manifiesto lo que quieren saber. Esos objetos son en este caso los homogéneos. Esto significa que se intenta llevar el fenómeno a condiciones extremas; aislar el mejor contexto en que aparece el problema. En este caso ¿qué podemos predicar acerca de la masa de un sonido? Ahora la masa de un objeto se ha tornado ella misma objeto de estudio (unidad de análisis) acerca de la cual vamos a predicar algo, conforme a criterios determinados. 
Ya identificado el tipo de masa de un sonido, en este caso fijo; si la masa es tónica o compleja, podremos ser un poco más sutiles en la percepción de la Masa.

Podremos apreciar una Textura de Masa.

Veamos cuales son:

Sonido Tónico. Es un solo "sonido". Por ejemplo el de un instrumento tradicional.

Grupo Tónico. Escucharemos no una, sino varias alturas bien definidas. Este es el caso del acorde. Estriado. Son sonidos ambiguos que según el contexto se percibirán como color o como intervalo. Por ejemplo las campanas de plancha los gongs etc.

Grupo Nodal. Son una variedad de alturas vagas. Por ejemplo varios temblores de platillos en distintas zonas del registro.

Sonido Nodal. Es una altura vaga o Masa compleja. Por ejemplo temblor de platillo en zona de tesitura.

\section{Timbre armónico}

Otro criterio es el Timbre Armónico. No hay que confundirlo con el timbre instrumental.

En los sonidos provenientes de instrumentos musicales, el timbre armónico corresponde al espectro de ese sonido (pensar en el timbre de las vocales). El espectro armónico no es en general el principal factor de reconocimiento de timbre instrumental. El timbre armónico es una cualidad de la materia sonora anexa a la masa. En los sonidos tónicos se puede distinguir muy bien un criterio de otro. En los sonidos complejos no es tan sencillo separar el timbre armónico de la masa.

\section{Tipos de timbre armónico}

Existen dos casos:

O bien la masa del sonido es una y el timbre armónico es también global, o la masa está dividida en distintas capas y al mismo tiempo puede, cada una de ellas poseer distintos timbres armónicos.

\section{Clases de timbre armónico}

Las clases de timbre armónico están vinculadas a las clases de masas:

Tanto el sonido puro como el ruido carecen de timbre; su timbre armónico es nulo. Para las masas tónicas el timbre es tónico.

Para los nodos, es decir grupo nodal y sonido nodal, el timbre es lo que no está descripto en la masa. Es algo así como un "resto". Este resto es susceptible de un análisis posterior. En este caso, hablamos de timbre complejo. Otras veces el timbre está totalmente soldado a la masa. Hablamos aquí de un timbre continuo o confuso. Es el caso de los platillos por ejemplo. Para los grupos tónicos el timbre puede ser tónico o continuo.

Para los estriados el timbre puede ser tanto complejo como continuo. 


\subsection{Criterio de mantenimiento}

\section{Grano}

Si bien lo clasificamos dentro de los criterios de mantenimiento, el grano constituye también una cualidad la materia sonora.

Podemos apreciar el grado de rugosidad del sonido tal como evaluamos el tipo de grano de la superficie de un objeto cualquiera.

Un sonido, debido al mantenimiento de un arco, una boquilla o un redoble de baquetas, puede comportar una microestructura que designamos con el nombre de Grano.

\section{Tipos de granos}

Digamos en principio que a los tres tipos de mantenimiento: continuo, nulo e iterativo, corresponden tres tipos de granos. Estos son los de frotamiento (Compactos), los resonancia (Armónicos) y los iterativos (Discontinuos).

\section{Clases de granos}

Es conveniente, escribe Schaeffer, hacer una descripción empírica de los tipos de grano tal como lo haríamos una superficie material (Schaeffer, 1988, p. 282). Se evalúa cómo se "escalona" cada tipo de grano (de resonancia, de frotamiento y de iteración).

Para hacer más compresible las clases de grano se hace necesario que lo hagamos conjuntamente con la descripción de los géneros de Grano.

Granos Compactos: un sonido de flauta presenta una banda de ruido muy apretada debido al aire necesario para mantener el sonido, que se encuentra bien diferenciada de su Masa. El grano de la flauta perceptivamente esta muy próximo al producido por el frotamiento de un arco.

Podríamos evaluar que lugar ocupa este género de grano en la escala que va desde lo rugoso a lo liso, pasando por el mate.

Granos Armónicos: Las notas graves de un piano presentan cierto centelleo. El sonido de un platillo, mas allá de su Masa y muy próximo al Timbre armónico, nos hace oír también una suerte de rápido hormigueo.

Podríamos evaluar que lugar ocupa este género de grano en la escala que va desde lo tembloroso a lo límpido, pasando por lo hormigueante.

Granos Discontinuos: el batido de la lengüeta de Fagot en las notas graves. Los redobles con baquetas duras y los sonidos muy graves en general, presentan un tipo de grano regular muy particular. Podríamos evaluar que lugar ocupa este género de grano en la escala que va desde lo grueso a lo fino pasando por lo cerrado.

En la práctica estos tipos de Granos se superponen y se combinan. Por ejemplo, una nota grave de piano posee tanto un tipo de Grano Armónico a causa de su mantenimiento, como un Grano discontinuo a causa de la zona del registro en que se localiza. 
Habría entonces géneros mixtos de granos:

Entre los armónicos y los discontinuos: Discontinuos armónicos (golpes resonantes, chirridos, machaqueos).

Entre los discontinuos y los compactos: Compactos discontinuos (redobles, zumbidos etc.). Entre los compactos y los armónicos: Compactos armónicos (frotamientos resonantes, crujidos murmullos.

\section{Marcha}

Si bien la marcha está agrupada en el criterio de mantenimiento, se puede considerar que la misma también es una cualidad de la forma. además de la percepción del perfil general del sonido, se puede apreciar cierta oscilación de nivel denominada marcha o movimiento.

El caso mas conocido es la resultante de una forma particular de mantenimiento denominada "vibrato". Sin embargo existen otros sonidos, carentes de mantenimiento, en los cuales es posible percibir esta oscilación de nivel. La marcha o movimiento seria, entonces, la generalización del concepto de "vibrato".

\subsection{Criterios que describen la forma}

\section{Perfil dinámico}

Michel Chión escribe que el criterio dinámico, bastante inconexo, reúne aquello que concierne a la intensidad del sonido y en especial a los ataques (Chion, 1999, p. 318).

En cuanto a la intensidad, el perfil dinámico representa la manera en ésta evoluciona en un objeto sonoro. Es necesario no confundir este criterio con la intensidad absoluta. Un sonido puede iniciarse bruscamente e ir progresivamente decreciendo en intensidad, o puede tener un forma delta, entre otras. No se contempla, entonces, el valor absoluto, es decir, cuan "forte" o "piano" es un sonido, porque esto depende de las condiciones de escucha. Por ejemplo, la fuente del sonido puede estar situada a mayor o menor distancia de quien escucha. Esto va a producir que escuchemos el sonido con mayor o menor intensidad. Ahora bien, lo que no va a cambiar es la variación de la intensidad del desarrollo del sonido. El "dibujo" que resulta de las variaciones de intensidad del desarrollo del sonido, es en gran medida independiente de las condiciones de escucha. Esto es lo que nos permite -dice Chion- “(...) reconocer el timbre de un instrumento en una grabación o en una retransmisión radiofónica, sea cual sea el volumen sonoro elegido" (Chion, 1999, p. 319). La percepción de la dureza del ataque esta en relación a la pendiente de disminución dinámica.

\subsection{Criterios que describen la variación de masa.}

Como ya se ha dicho en este artículo, Schaeffer reserva dos criterios especiales para describir los casos en que la masa de un sonido es variable. Uno de esos criterios es el perfil melódico, el otro el Perfil de Masa. 


\section{Perfil melódico}

Este criterio da cuenta de aquellos sonidos en los cuales la masa evoluciona en el campo de las alturas. Es decir cuando varía su tesitura.

Estas variaciones pueden ser discontinuas o continuas.

Un claro ejemplo de variaciones discontinuas es la melodía. La melodía en este caso es concebida como un objeto musical variado y no como la articulación de diferentes notas.

Schaeffer en su intento de justificar la noción de perfil melódico de se remite a la historia de la escritura musical y en especial a la escritura neumática. Los neumas reflejaban los movimientos de la voz (excluyendo el valor de los intervalos, de la altura absoluta y las duraciones) y los gestos "quironómicos" "cuando la melodía presentaba melismas de dos, tres o cuatro notas sobre una misma sílaba". Schaeffer concluye que durante varios siglos la unidad musical fue el "objeto musical variado y no su descomposición en notas" (Schaeffer, 1988, pp. 288-289).

Estas variaciones sin duda están ligadas también al acento como al ritmo, “(...) así pues, los neumas han asociado varios rasgos y creado figuras plenas de sentido, surgidas de una morfología evolucionada" (Schaeffer, 1988, pp. 288-289)

Luego se recurrió a letras para expresar valores y a neumas para representar objetos. Cuando esta escritura, por razones de operatividad, dio lugar a la escritura actual, subsistieron sin embargo las ligaduras de expresión.

Pierre Schaeffer dice que existe un profundo conflicto entre dos maneras de representar los objetos musicales y concebir la música. Es decir la continuidad de las figuras (el gesto, identidad entre acción y percepción), y la discontinuidad de los valores métricos e interválicos.

Esta distinción entre continuidad de las figuras y discontinuidad de los valores la encontramos en algunos trabajos de investigación acerca del desarrollo de la aprehensión de nociones musicales en el niño. Algunos de estos trabajos son realizados a partir de las escrituras musicales espontáneas de los niños, en especial, haciendo hincapié en el ritmo y la melodía (Bamberger, en Sinclaire, 1988).

\section{Perfil de masa}

Este criterio esta referido a las variaciones de la "anchura", es decir las variaciones que afectan al calibre de la Masa.

Es decir, la forma resultante de las variaciones de anchura de la masa.

\section{Consideraciones finales}

Como habrá advertido el lector, se hace un tanto difícil comprender los conceptos de la tipología y la morfología sin ejemplos sonoros que los ilustren. El solfeo del objeto sonoro publicado por Schaeffer con posterioridad a la primera edición del Tratado... tuvo como propósito saldar esa deuda (Schaeffer y Reibel, 1988). Sin embargo, como ya he dicho más arriba, ese trabajo, ilustra de manera satisfactoria todos los casos incluidos en la Tipología pero no así los del cuadro morfológico. Queda pendiente la publicación de ejemplos sonoros que den cuenta de todos los casos pertenecientes a la morfología. 
Pero existe otra dificultad. La tipología y la morfología de los objetos sonoros es sólo una parte del trabajo de Schaeffer. Y para comprenderla cabalmente se hace necesario abordar otros conceptos que figuran en su Tratado.

Encuentro en el Tratado de los objetos musicales cuatro conceptos cuya articulación en la escritura de Schaeffer apenas quedó esbozada.

Estos cuatro conceptos son, a mi entender, el material a partir del cual se pueden articular y organizar los términos de una teoría perteneciente a una nueva ciencia que Schaeffer denominó "Aculogía". 9

Coexisten en el tratado una Metafísica; una teoría de las escuchas; una teoría del objeto de esa escucha y una teoría del valor.

Lo que llamo metafísica schaeffereana, está representada por el golpe que Schaeffer asesta a la psicofísica de su tiempo. En especial al descubrimiento de que no hay una correspondencia término a término entre las señales físicas y los datos perceptivos. De allí se infiere en Schaeffer una metafísica en el sentido de que esboza un "perfil general de las relaciones del hombre con el mundo". Pero además, es una metafísica puesto que el Tratado de los objetos musicales se ubica literalmente "más allá de la física" (o más acá, según se lo mire).

La teoría de las escuchas en este artículo ha quedado apenas señalada. Pero además, las conclusiones de Schaeffer respecto de las escuchas está a la espera de nuevos aportes, en especial, de la semiología.

Lo que en este artículo se ha resumido es parte de la teoría del objeto. Pero es sólo eso, una parte. Es lo más conocido de la obra de Schaeffer, además de las tres escuchas. Pero la cuestión del objeto sonoro es mucho más compleja. La publicación castellana de su Tratado es una versión reducida. Hay aspectos de la teoría del objeto sonoro que han quedado mutilados. En especial la dialéctica del objeto-estructura.

La teoría del valor tiene como propósito indagar cuáles son los estados posibles que un sonido adquiere a partir de su marco de referencia en una composición musical. ${ }^{10}$ En otras palabras, toda música implica un género de objetos musicales, esto es, objetos que comparten características comunes y en virtud de ello, hacen posible la emergencia de cierto valor.

La música de la tradición occidental se ha conformado con objetos cuyo carácter

fundamental es su alto grado de tonicidad, al tiempo que sus otras variables quedan relativamente neutralizadas. Esto posibilita la percepción de las diferencias de altura y en consecuencia la construcción de un determinado tipo de sistema de referencia.

Cada sistema musical presupone, por lo tanto, la elección de cierto género de objetos musicales. Es posible confrontar objetos de un mismo género (distintos de los objetos utilizados por la tradición) para verificar qué valores ponen de relieve.

Schaeffer no es sólo un autor. Y el Tratado de los objetos musicales no es una mera "fonética de los ruidos", como algunos pretenden. Schaeffer es un productor de discursividad. Las preguntas que se formuló marcan, a mi juicio, un antes y un después respecto del estudio del sonido. Si se quiere avanzar en esta línea de investigación se hace necesario retornar una y otra vez a las preguntas que Schaeffer inauguró. Retomar esas preguntas es una condición necesaria para quienes estamos interesados en ir en busca de lo audible. 


\section{Notas}

1. El concepto de escucha reducida hace referencia a la "reducción fenomenológica", de Edmund Husserl. Veasé Libro III "Objetos y estructuras" del Tratado de los objetos musicales pp. 159-169.

2. El presente trabajo es una guía para orientar la comprensión de la taxonomía Schaeffereana. En especial, el texto estará basado en El Libro IV Morfología y tipología de los objetos sonoros y solfeo de los objetos musicales, perteneciente al Tratado de los objetos musicales en su versión española. pp, 215- 294.

3. Pierre Schaeffer y Guy Reibel. Solfege de l’objet sonore. Paris , Buchet/Castel. 1998. Esta obra contiene tres discos compactos con los ejemplos sonoros. Además esta edición de 1998 está traducida al castellano.

4. Estas son las primeras tres operaciones. Las otras son la caracterología, el análisis y la síntesis. 5. Sólo doy ejemplos de los casos pertenecientes a instrumentos musicales. Faltaría dar ejemplos de otras cadenas sonoras. El lector se encontrará con los recortes de objetos de todas las cadenas sonoras en: Pierre Schaeffer y Guy Reibel.1998. op.cit. ,pp 149-151, y en los ejemplos sonoros 14 al 22 del CD 3, adjuntado a esa obra.

6. Para una mayor comprensión de la tipología se hace necesario que el lector disponga, al menos, de la versión castellana del Tratado de los objetos musicales. Para el caso de la Tipología, y con el propósito de comprender cómo Schaeffer organiza el cuadro clasificatorio, en la página 242 se encontrará con el "cuadro recapitulativo de la tipología", que por razones de espacio no es posible reproducirlo en el presente trabajo.

7. Volveré sobre este tema en el apartado que describe la morfología.

8. Schaeffer realiza un cuadro de doble entrada- una suerte de tabla de los elementos- donde cruza los siete criterios morfológicos (en las filas) con las categorías de tipo, clase, género y especie (en las columnas). El lector se encontrará con este "Cuadro de recapitulación del solfeo de los objetos musicales” en las páginas 290 a 293 del “Tratado...”. En este artículo sólo trabajaremos con las columnas que se refieren a los tipos y a las clases. Sin embargo es indispensable tenerlo a mano.

9. El término "Aculogía" fue acuñado por Schaeffer para designar una ciencia cuyo propósito es estudiar " aquello que el sujeto oye".

10. Si la teoría del objeto sonoro representa las relaciones "inter- objetos". Es decir los tipos y clases de percepción , cualificación y diversificación de los criterios del objeto. La teoría del valor representa las cuestiones trans- objetos, ubicadas al el final del cuadro. Schaeffer indaga la relación que los objetos mantienen con las estructuras musicales. Es decir: el "salto" de lo sonoro a lo musical ( ver Schaeffer; 1988: 292). 


\section{Lista de Referencias Bibliográficas}

Banberger, J. (1988). Les structurations cognitives de l'appréhension et de la notation de rytmes simples. En Sinclair, H. (ed). La producción de notations ches le Jeune enfant. Paris: Press Universitaires de France.

Chion, M. (1999). El sonido: Música, cine y literatura... Barcelona: Paidós.

(1993). La audiovisión: Introducción a un análisis conjunto de la imagen y el sonido. Barcelona: Paidós.

(1983). Guide des objets sonores: Pierre Schaeffer et la recherche musicale. Paris: Buchet/Castel. Delalande, F. (1995). La música es un juego de niños. Buenos Aires: Ricordi.

Dolar, M. (2007). Una voz y nada más. Buenos Aires: Manantial.

Saitta, C. (2002). El diseño de la banda sonora en los lenguajes audiovisuales. Buenos Aires: Saitta publicaciones musicales.

Schaeffer, P. (1988). Tratado de los objetos musicales. Madrid: Alianza.

(1966). Traité des Objets Musicaux: essai interdisciplines. Paris. Éditions du Seuil.

(1959). ¿Qué es la música concreta? Buenos Aires: Nueva visión.

Schaeffer, P. y Reibel, G. (1998). Solfege de l'objet sonore. Paris: Buchet/Castel.

Summary: The present article aims to facilitate the understanding of the Typology and the Morphology of the sonorous objects, including in the famous Treaty of the musical objects by Pierre Schaeffer. In the first place the exploratory phase of the construction of the taxonomy of the sounds is described, that Schaeffer denominated Type morphology. Secondly the nine cases of central Typology appear. And finally the seven morphologic criteria are explained.

Key words: morphology - sonorous objects - typology.

Resumo: O artigo tem o objetivo de faciliar a comprensão da Tipologia e a Morfologia dos objetos sonoros, incluida no célebre "Tratado dos objetos musicais" de Pierre Schaeffer.

No primeiro lugar se descreve a fase exploratoria da construcção da taxonomia dos sons, que Schaeffer chamou Tipo - Morfologia. No segundo lugar se apresentan os nove casos de Tipologia central. E por último se explicam os sete critérios morfológicos.

Palavras chave: morfologia - objetos sonoros - tipologia. 